\title{
Correction to: Retinal pathology in experimental optic neuritis is characterized by retrograde degeneration and gliosis
}

Praveena Manogaran ${ }^{1,2^{*}}$, Marijana Samardzija ${ }^{3}$, Anaïs Nura Schad ${ }^{4}$, Carla Andrea Wicki ${ }^{2,5}$, Christine Walker-Egger ${ }^{2}$, Markus Rudin ${ }^{1,6,7}$, Christian Grimm ${ }^{3}$ and Sven Schippling ${ }^{2}$

\section{Correction to: Acta Neuropathol Commun (2019) 7:116 https://doi.org/10.1186/s40478-019-0768-5}

In the original publication of this article [1], figure 10 contained two panels " $\mathrm{C}$ " as panel " $\mathrm{F}$ " was accidentally omitted. The incorrect (Fig. 1) and correct (Fig. 2) versions are published in this correction article.

\begin{abstract}
Author details
${ }^{1}$ Department of Information Technology and Electrical Engineering, Swiss Federal Institute of Technology, Zurich, Switzerland. ${ }^{2}$ Neuroimmunology and Multiple Sclerosis Research, Clinic for Neurology, University Hospital Zurich and University of Zurich, Zurich, Switzerland. ${ }^{3}$ Department of

Ophthalmology, Lab for Retinal Cell Biology, University of Zurich, Zurich, Switzerland. ${ }^{4}$ Department of Biology, University of Zurich, Zurich, Switzerland. ${ }^{5}$ Department of Health Sciences and Technology, Swiss Federal Institute of Technology, Zurich, Switzerland. ${ }^{6}$ Institue for Biomedical Engineering, Swiss Federal Institute of Technology and University of Zurich, Zurich, Switzerland. ${ }^{7}$ Institute of Pharmacology and Toxicology, University of Zurich, Zurich, Switzerland.
\end{abstract}

Published online: 18 October 2019

\section{Reference}

1. Manogaran P, Samardzija M, Schad AN et al (2019) Retinal pathology in experimental optic neuritis is characterized by retrograde degeneration and gliosis. Acta Neuropathol Commun 7:116. https://doi.org/10.1186/s40478019-0768-5

\footnotetext{
* Correspondence: pmanogar@student.ethz.ch

${ }^{1}$ Department of Information Technology and Electrical Engineering, Swiss Federal Institute of Technology, Zurich, Switzerland

${ }^{2}$ Neuroimmunology and Multiple Sclerosis Research, Clinic for Neurology,

University Hospital Zurich and University of Zurich, Zurich, Switzerland

Full list of author information is available at the end of the article
}

(c) The Author(s). 2019 Open Access This article is distributed under the terms of the Creative Commons Attribution 4.0 International License (http://creativecommons.org/licenses/by/4.0/), which permits unrestricted use, distribution, and reproduction in any medium, provided you give appropriate credit to the original author(s) and the source, provide a link to the Creative Commons license, and indicate if changes were made. The Creative Commons Public Domain Dedication waiver (http://creativecommons.org/publicdomain/zero/1.0/) applies to the data made available in this article, unless otherwise stated. 


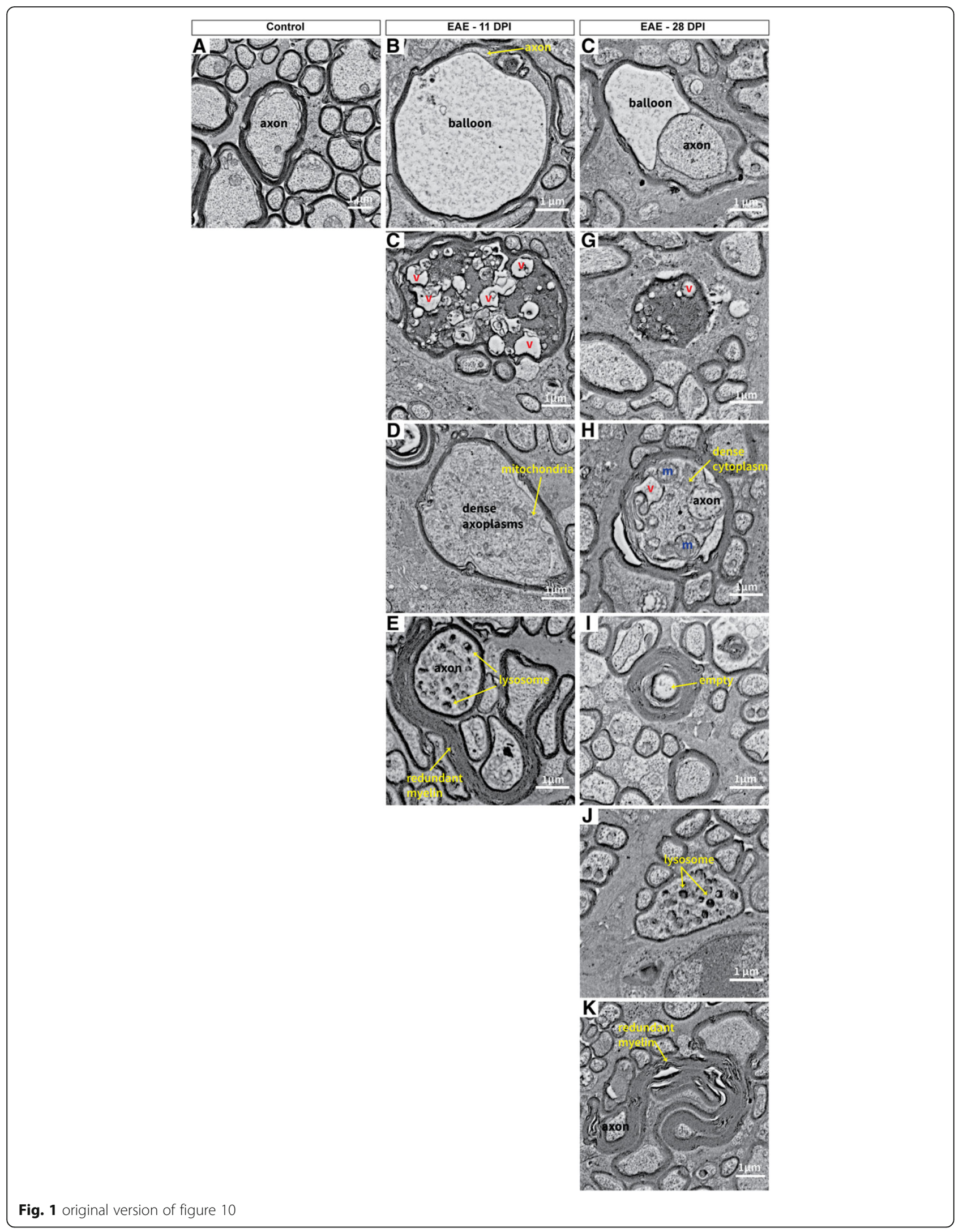




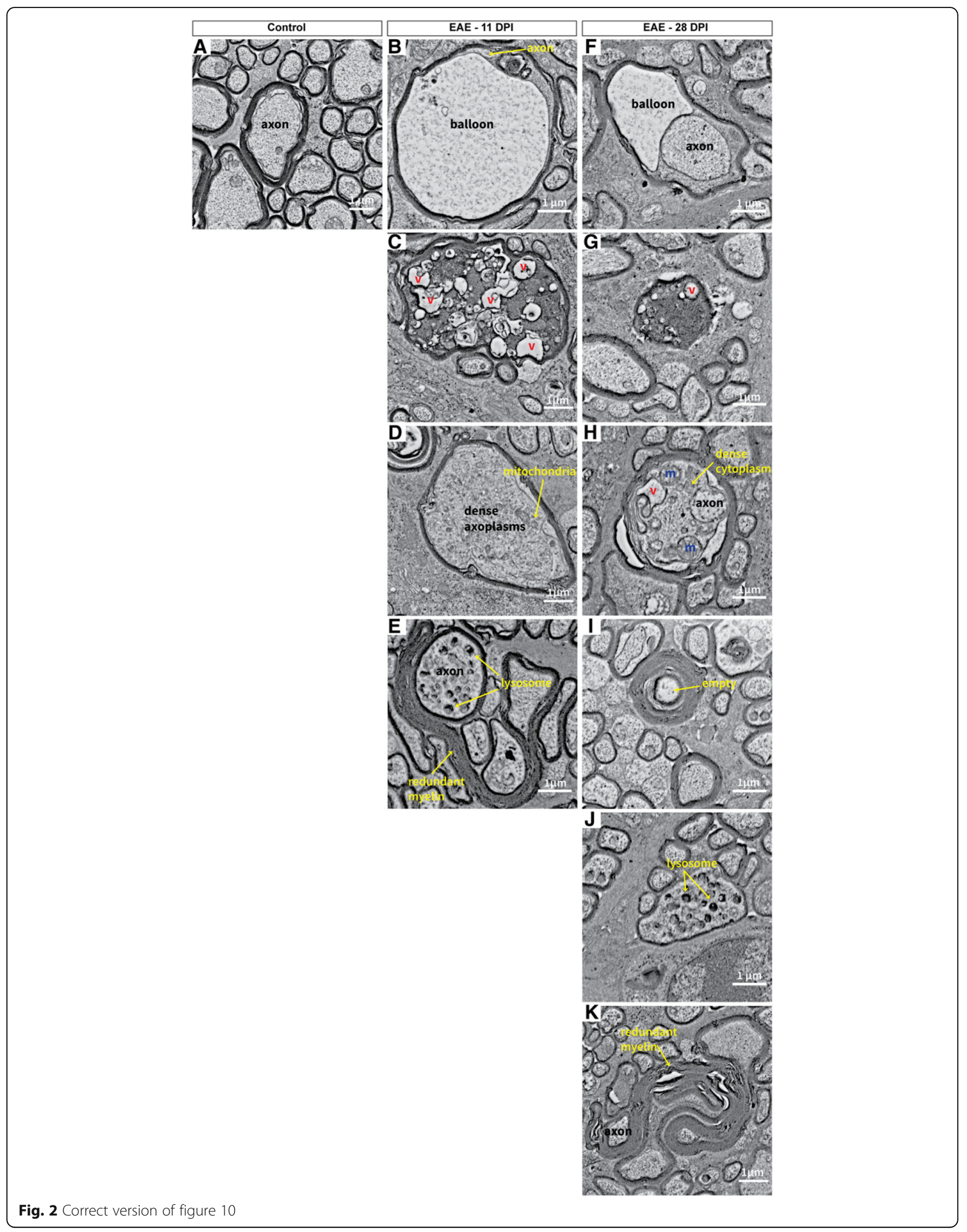

